\title{
Towards Transnational Interoperable PPDR Communications: the European ISI Cloud Network
}

\author{
Ramon Ferrús, Oriol Sallent \\ Signal Theory and Communications Department \\ Universitat Politècnica de Catalunya (UPC) \\ Barcelona, Spain \\ [ferrus, sallent]@tsc.upc.edu
}

\author{
Kirsten Aabye, Aladdin Rashed \\ Architecture Research Board \\ Motorola Solutions \\ Copenhagen, Denmark
}

[kirsten.aabye, aladdin.rashed]@motorolasolutions.com

\author{
Claudia Olivieri \\ System Design Engineer \\ Selex ES \\ Genova, Italy \\ claudia.olivieri@selex-es.com
}

\author{
Franco Pangallo, Debora Proietti Modi \\ Radio Mobile Department \\ Istituto Superiore delle Comunicazioni (ISCOM) \\ Rome, Italy \\ [franco.pangallo,debora.proiettimodi.ext]@mise.gov.it
}

\begin{abstract}
The European Council has been stressing the need for interoperability among technologies used for Public Protection and Disaster Relief (PPDR) communications across Europe for a long time. Nevertheless, while the introduction of TETRA and TETRAPOL technologies in the last two decades has increased the possibility to talk cross agency internally in a country, cross border communication for the public safety forces is not well solved as of today. This paper describes the communications interoperability solution that is being developed in the framework of the ISITEP project. This solution, referred to as the European Inter-System Interface (ISI) Cloud Network, aims to integrate the PPDR national/regional infrastructures to allow migration (i.e., roaming) and communication services between networks within a secure framework. The ISI Cloud Network involves, among other components, the specification of a new ISI interface to be deployed over IP transport networks and the development of a number of different gateways to cover the use of TETRA and TETRAPOL technologies as well as the use of legacy TETRA ISI by some networks.
\end{abstract}

Keywords - Inter-System Interconnection, TETRA, TETRAPOL, Public Safety Communications, Emergency Services Communications

\section{INTRODUCTION}

The Public Protection and Disaster Relief (PPDR) sector brings essential value to society by creating a stable and secure environment to maintain law and order and to protect the life and values of citizens. PPDR services such as law enforcement, firefighting, emergency medical services and disaster recovery services are pillars of our society organisation. The PPDR sector is for most nations intimately connected to the public sector of society, either directly as part of the governmental structure or as a function with is outsourced under strict rules and intensively monitored by government's contracting ministry or department. The most important part of the PPDR work is done in the field. Therefore, secure radio communications are extremely important to PPDR organizations. Indeed, at times, radio communication is the only form of communications available.

A major limitation associated with PPDR systems and the arrangements used nowadays in emergency and disaster relief scenarios (see e.g., [1]) is the lack of communications interoperability: the diversity of Private/Professional Mobile Radio (PMR) technologies (e.g., TETRA, TETRAPOL, P25, DMR, analog VHF radios) used by PPDR organisations often inhibits cooperation between different agencies. Moreover, even when using the same technology, PPDR communications might be provided to different PPDR agencies through independent, separated networks that are not typically interconnected for service interworking.

While this interoperability barrier in many European countries has been partially addressed at national level through the deployment of large scale TETRA or TETRAPOL shared networks that serve the needs of the different PPDR agencies within a country [2], transnational interoperability between these PPDR networks still remains an issue as of today [3]. The growth of international crime (e.g., drugs, human trafficking) requires joint police operations on the field in areas such as cross-border pursuit of criminals and crossborder patrols and controls. The need of cooperation is also

Article accepted for publication by IEEE.

DOI: 10.1109/ICT-DM.2015.7402044

For citation use the following:

R. Ferrús et al., "Towards transnational interoperable PPDR communications: The European ISI Cloud Network," 2015 2nd International Conference on Information and Communication Technologies for Disaster Management (ICT-DM), Rennes, 2015, pp. 96-102. doi: 10.1109/ICT-DM.2015.7402044. URL:

http://ieeexplore.ieee.org/stamp/stamp.jsp?tp=\&arnumber=7402044\&isnumber $=7402014$

(C) 2015 IEEE. Personal use of this material is permitted. Permission from IEEE must be obtained for all other uses, in any current or future media, including reprinting/republishing this material for advertising or promotional purposes, creating new collective works, for resale or redistribution to servers or lists, or reuse of any copyrighted component of this work in other works. 
growing in the last decade in natural calamities (flooding, earthquakes etc.), disasters (like bomb attacks aircraft crashes, chemical nuclear alert, etc.) and generally for injured care and transportation, fire fighting and support for civil protection. Since time is critical in disaster relief, international cooperation enables a greater effectiveness. Such strategy has been proving to be effective as for example between Norway and Sweden that are sharing cross border resources to combat fire or to transport patients. Effective interoperability may greatly reduce such damages if PPDR resources can rapidly operate in foreign areas. In this context, the relevance of cross border security operations is already acknowledged at European level and identified as a priority (Schengen Agreements). In addition, according to the article 222 of the Treaty of Lisbon ("mutual solidarity"), the European Union (EU) shall mobilize Member States resources to assist other Member States in case of terrorist attacks or in case of natural/man-made disasters.

Thus far, the common ways to establish communications between countries are mainly based on the exchange of radio terminals and the use of radio gateway solutions [4]. By exchanging radio terminals, foreign PPDR officers could operate over the entire visited country. However, communications with their own country are not possible through the loaned terminal, except if they bear a second terminal from their own network and if operations are still within the coverage of their own national network (e.g., common cross border patrols with radios from both networks in the car). On the other hand, radio gateways solutions are used for the interconnection of the audio signals between separate networks through the deployment of back-to-back radios. The main advantage of this solution is that the first responders can use their own usual terminals to talk to users from other countries (e.g., talk groups in the two networks are patched by the radio gateways). However, first responders cannot use their terminals on another network abroad (no "roaming" capability): everybody has to remain under the coverage of its own national radio network.

Specific groups of countries (e.g., France-Switzerland, Norway-Sweden, Sweden-Germany, Belgium-Netherlands) are recently cooperating to address communications interoperability for PPDR cross-border operations [3][4]. Nevertheless, without an international solution, interoperability will be possible only in localised areas, thus vanishing the benefits of an extensive cooperation. In addition, there is a growing demand of standard interoperability solutions by industry, since new international tenders require multivendor interoperability.

In this context, the European research project ISITEP [5] is an ambitious initiative aimed at developing procedures, technology and legal agreements to achieve an effective solution for PPDR interoperability across European countries. End users participating in ISITEP have driven the requirement to guarantee legal, operational and technical coherence. ISITEP will demonstrate full radio interface migration for PPDR resources in diverse scenarios such as Norway-Sweden border, Germany-Belgium-Holland border and Swiss-French border.

Within the ISITEP framework, this paper describes the communications interoperability solution that is being developed. This solution is referred to as the European InterSystem Interface (ISI) cloud network. To this end, the rest of the paper is organized as follows. Section II provides an overview of the architecture reference model and interconnection configurations. Section III describes the specifications of the new interfaces and network gateways under development as well as the connectivity options being considered for the realisation of the European ISI cloud network. Section IV analyses the extension of the interoperability solution beyond current TETRA/TETRAPOL services, especially considering the future adoption of mobile broadband capabilities (e.g., Long Term Evolution [LTE] access) in future PPDR networks. Finally, conclusions are drawn in Section V.

\section{OVERVIEW OF THE EUROPEAN ISI CLOUD NETWORK}

The European ISI cloud network is intended to integrate the PPDR national/regional infrastructures to allow migration (i.e., roaming) and communication services (e.g., individual and group calls, short data services and applications on top of them) between networks within a secure framework. Its architecture reference model and intended interconnection configurations are described in the following.

\section{A. Architecture reference model}

The architecture reference model for the European ISI Cloud Network is depicted in Figure 1. The model encompasses a set of functional components involved in the interconnection of two TETRA or TETRAPOL networks and the interfaces/reference points among the functional components.

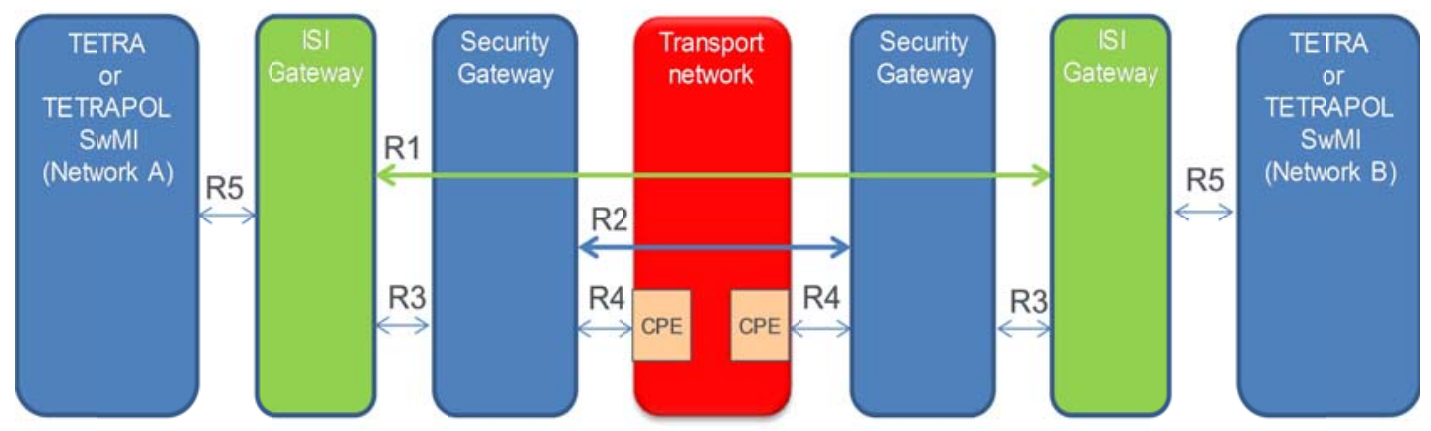

Fig. 1. Architecture reference model of the ISI cloud network 
The functional components within the architecture reference model are the following:

-ISI Gateway. Element that provides the Inter-System Interface (ISI) functionalities for the interconnection of TETRA and TETRAPOL networks. A number of different ISI Gateways have been defined to support the different combinations of radio technology (TETRA, TETRAPOL) and interconnection technology (E1 circuits, IP transport).

-Security Gateway. Element that provides enhanced protection to traffic and signalling information running on the interfaces that cross PPDR network operator boundaries.

-Transport network. External network for the E1/IP interconnection (international links) of the national PPDR networks.

-TETRA/TETRAPOL switching and management infrastructure (SwMI). This represents the core functionality of existing PMR networks.

The following interfaces/reference points are defined between the above components:

$-R 1$ : Reference point between two remote ISI gateways. Two protocol stacks are under consideration for the implementation of this reference point: the existing ETSI TETRA ISI [6], which is based on circuit-switched technologies, and a new ISI over IP interface being specified in ISITEP, which is based on IP technologies. Both protocol solutions are based on a pointto-point service model (i.e., the R1 reference point terminates between two peer ISI gateways). It is considered that SwMIs do not perform transit-switching functions.

$-R 2$ : Reference point between two remote Security Gateways. It will enable the support of the essential security requirements needed to interconnect national networks within the interoperability cloud. It could rely on the use of protocols such as the IPsec protocol in tunnel mode in case of IP transport connectivity. A point-to-point service model is also assumed in this interface (i.e. a R2 reference point terminates between two peer Security Gateways).

-R3: Reference point between the ISI Gateway and the Security Gateway. This interface is intended to allow the implementation of the ISI Gateway and the Security Gateway in separate physical devices. An internal packet-switched network (e.g., Ethernet) could be used for the interconnection of these two elements.

$-R 4$ : Reference point between the Security Gateway and the Customer Premise Equipment (CPE) of the transport network used for international interconnection. The interface to the CPE depends on the transport technology. In the case of IP interconnection, this interface can be e.g. a standard Ethernet interface.

-R5: The Interface between the TETRA or TETRAPOL SwMI and the ISI gateway. The implementation of this interface is technology/vendor specific. In the case of TETRA, it could be based on IP or circuit-switched technology. In the case of TETRAPOL, the Call Control (CC) Application Programming Interface (API) Server interface could be used.

\section{B. Interconnection configurations}

Different interconnection configurations are envisioned based on the following dimensions:
-Technology used in the radio interface and in the ISI interface of the interconnected networks. This basically is related to the implementation of reference points R1 and R5.

-Number of interconnected networks. This basically depends on the service model used in R1 and R2.

As to the first dimension, five different interconnection configurations (illustrated in Figure 2) are identified when considering the different possible implementations of R1 and R5 reference points:

-Configuration A. Two TETRA networks are interconnected using the R1 implementation based on ISI over IP. Internally, these networks can use either IP-based or circuit-switchedbased interfaces in the R5 reference point between the SwMI and the ISI Gateway.

-Configuration B. Two TETRA networks are interconnected and one of them provides support for the ETSI TETRA ISI interface.

-Configuration C. Interconnection of TETRA networks based on the ETSI TETRA ISI interface.

-Configuration D. Interconnection of TETRA and TETRAPOL networks. Protocol ISI over IP is used in the R1 reference point. R5 in the TETRA network can be either packet or circuit-switched based. R5 in the TETRAPOL is based on the Call Control (CC) API Server.

-Configuration E. Interconnection of TETRAPOL networks. ISI over IP gateways are Protocol ISI over IP is used in the R1 reference point. R5 is based on the TETRAPOL Call Control (CC) API Server.

Interfaces R2, R3 and R4 do not impact on the above classification. However, the implementation of the interface $\mathrm{R} 2$ and R3 is directly related to the solution adopted for R1.

As to the dimension considering the number of networks to be interconnected, given that R1 and R2 reference points are both based on a point-to-point service model, the interconnection of PPDR networks shall be done in pairs.

\section{EUROPEAN ISI CLOUD NETWORK SPECIFICATION}

A description of the key components (new ISI over IP interfaces, ISI gateways, Security Gateways and transport connectivity options) needed for the realisation of the European ISI cloud network is addressed in the following.

\section{A. New ISI over IP interface}

ETSI started the standardization process for TETRA ISI to support cross-border cooperation between independent TETRA networks in the 1990s. The TETRA ISI application is built on top of the PSS1 protocol stack for interconnecting Private Integrated services Network eXchanges (PINXs) to form Private Integrated Services Network (PISN) [7]. PSS1 is the ISO term; the PSS1 protocol is also known, informally, as QSIG, referring to reference point ' $Q$ ' defined by the European Computer Manufacturers Association (ECMA) [8], which developed most of the signalling protocols comprised in the PSS1 protocol. 
(A)

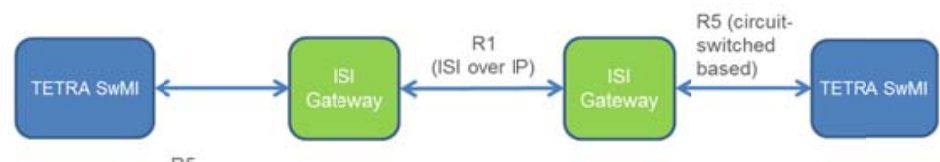

(B)

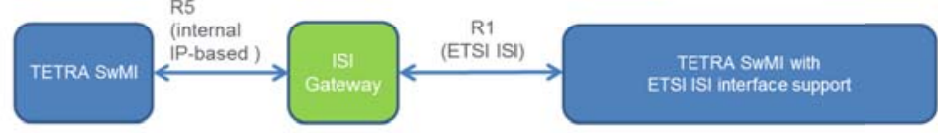

(C)

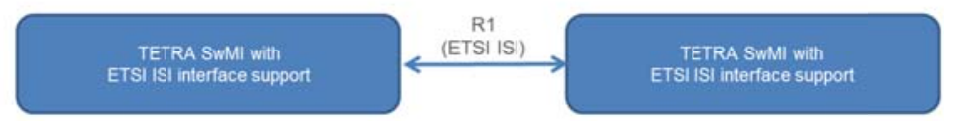

(D)

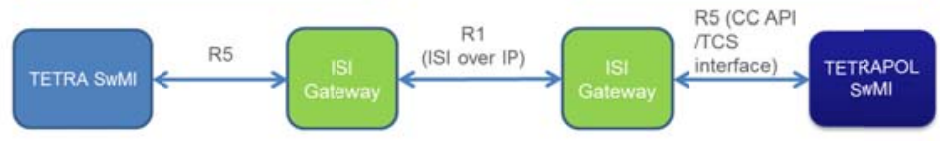

(E)

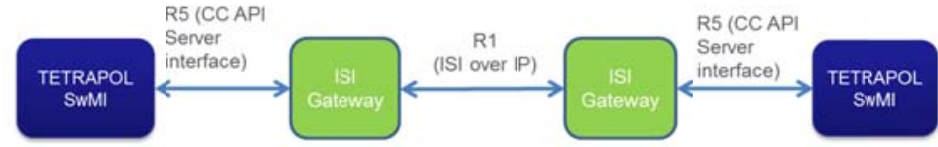

Fig. 2. Interconnection configurations based on the different possible implementations of R1 and R5 interfaces

A simplified view of the TETRA ISI protocol stack is depicted in Fig. 4. The TETRA ISI functionalities are organised in the following set of so-called Additional Network Features (ANFs):

- Additional Network Feature - ISI Individual Call (ANF-ISIIC);

- Additional Network Feature - ISI Group Call (ANFISIGC);

- Additional Network Feature - ISI Short Data service (ANF-ISISDS); and

- Additional Network Feature - ISI Mobility Management (ANF-ISIMM).

The signalling needs for TETRA ISI operation, which are not directly supported by PSS1/QSIG protocols, are provided by ISI Generic Functional Protocol (GFP). ISI GFP does not by itself control any ANF-ISI Protocol Data Units (PDUs) but rather provides a means to convey them. The Remote Operation Service Element (ROSE) is used to convey ANFISI PDUs. The Segmentation Service Element (SSE) is used for segmentation of long messages. For speech transmission, the TETRA coded speech frames are carried in $64 \mathrm{kbit} / \mathrm{s}$ E1 channels. The general aspects of the ISI are specified in ETSI EN 300 392-3-1 [6], which provides pointers to a number of specifications covering the individual ANFs. Today, such a standard is employed only by a few TETRA vendors for limited functionalities (i.e., mobility management, group call, individual call and short data).

The currently most used standard for Voice over IP (VoIP) communications is the Session Initiation Protocol (SIP), which may be conveniently used for the implementation of the proposed new ISI interface over IP transport. The approach adopted in ISITEP is to change the ETSI ISI ANFs so that they become bearer protocol independent. In this way, SIP could be used as a bearer protocol instead of QSIG. The current ETSI ISI protocols make use of the QSIG channel allocation for control of audio transport. For ISI over IP the Session Description Protocol (SDP) will be used for controlling the audio path. Hence, the SIP protocol would be used to convey SDP messages and ISI messages between SwMIs. This approach is illustrated in Figure 4, where it is also shown that voice traffic will be now supported over the Real-time Transport Protocol (RTP). The SIP protocol may be conveyed over UDP or TCP dependent on bilateral agreements. This IP-based implementation of the ISI application is referred to as "ISI over IP".

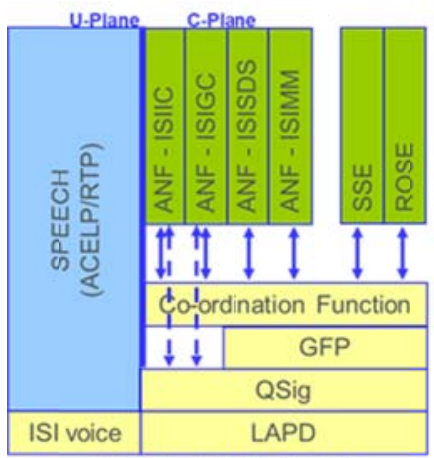

ETSI TETRA ISI Model

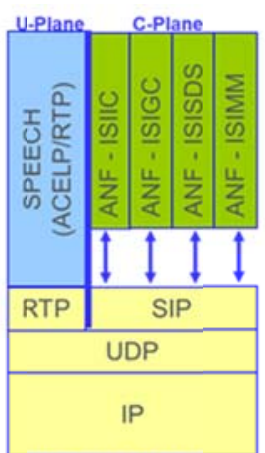

ISI over IP Model
Fig. 4. Existing ETSI TETRA ISI and proposed ISI over IP models

ISITEP at this aim will provide, at TETRA-TETRAPOL network level, public specifications to be subdued to the ETSI standard. Such public specifications will be produced by all the European network manufactures with the approval of relevant operators and end users. ETSI standardization cannot be achieved during ISITEP time frame because such process is normally longer than three years on these topics. In any case, 
since ISITEP public specifications will be demonstrated and approved on the field by end users and operators, the following standardization process is expected to be shortened submitting a Public Available Specification (PAS) to ETSI.

\section{B. Gateway}

Based on the possible interconnection configurations discussed above, three different ISI Gateways have to be developed in ISITEP (depicted in Figure 5):

-GW1: Gateway that allows the interconnection of existing TETRA networks (based on Motorola and SELEX ES equipment) through the new ISI over IP protocol.

-GW2: Gateway that provides translation between the QSIG / E1 and SIP / IP in both directions. The objective is to allow TETRA networks that have been implemented on circuit switched based technology to interoperate with packet switched implementations of TETRA.

-GW3/4: Gateway allowing a TETRAPOL network via Call Control (CC) API to be interconnected with another TETRA or TETRAPOL network through the new ISI over IP protocol. In particular, GW3 would allow for the interconnection of a TETRA and a TETRAPOL Regional Network, through a CC API / TCS interface for signalling and analog or S0 digital audio signal for voice. GW4 would allow for the interconnection of TETRAPOL to TETRAPOL Regional Networks, via CC API interface for signalling and analog or S0 digital audio signal for voice.

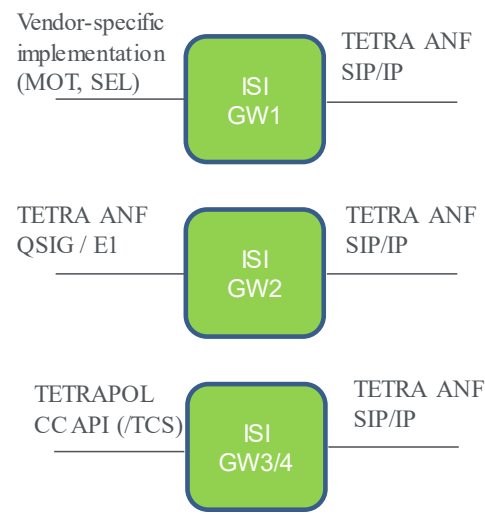

Fig. 5. Variants of the ISI Gateways to be developed in ISITEP

\section{Security gateway}

The security gateway delivers enhanced protection to traffic and signalling information running on the interfaces that cross PPDR network operator boundaries. The Security Gateway provides two basic groups of functions:

-Confidentiality and integrity of traffic exchanged among networks. The technical solution to provide required security of connections will be defined. This may include use of commercial encryption and Virtual Private Network (VPN) solutions as well as extension of some country specific (high) security connections to the connected network interface. The link security must be ensured not to degrade the capacity and QoS of the interconnections.

-Prevention of intrusions into the national networks. The existing TETRA and TETRAPOL networks have national security requirements, which must be fulfilled at the points of international interconnections. The task will define the technical solutions at the interconnection points to fulfil those requirements. The requirements may vary, depending on using commercial connectivity services versus direct secured links between the two parties, preventing intrusion.

\section{Transport network}

Two main options can be distinguished for the deployment of the new ISI over IP interface between PPDR networks:

-Direct IP Interconnection, in which the interconnection is established directly between the PPDR operators' networks, using either self-deployed transport capacity or leased connectivity services from a third-party network.

-Indirect IP Interconnection, in which the interconnection between operators' networks uses an IP Interconnection Intermediate Carrier, that is, a third party carrier that specifically provides IP Interconnection services in different levels. The concept of IP Interconnection service should be understood here as an interconnection service whose scope goes beyond the pure technical/network layer scope and takes into account the requirements for the services supported by that interconnection (e.g., IP Interconnection service for ISI services, IP Interconnection service for VoIP services). Hence, in addition to IP connectivity, IP Interconnection services might provide additional functions such as Domain Name Services (DNS), interworking functionality (e.g., transcoding) and service-level functionality (e.g., service-level proxies) [9].

In the case of Direct IP Interconnection, the relationship between the operators is bilateral. While this model would allow PPDR operators to have a complete control over the IP Interconnection, it may not be efficient for most inter-operator connections owing to the cost and complexity of maintaining individual interconnections with a high number of operators. However, direct connections may be still the option of choice for the interconnection a given pair of PPDR networks with specific interconnection requirements and conditions (e.g., high capacity needs between two networks that make it preferable to go for this model). Transport capacity between the two networks can rely on the use of any sort of connectivity service (e.g., Layer 1 such as PDH/SDH, Layer 2 such as Carrier Ethernet, Layer 3 such as IP VPN) for the transport of IP traffic between the networks (e.g., selfdeployed connectivity provided by any of the involved operators or leased lines rented from a third party intermediate carrier network).

In the case of Indirect IP Interconnection, a single IP Interconnection Intermediate Carrier Network could be used to provide interconnection service to a number of PPDR networks (in contrast to relying only on bi-lateral direct interconnections). Moreover, this IP Interconnection Intermediate Carrier Network could offer additional functionality (e.g., service awareness, destination routeing, accounting services, etc.). An approach that fits under this 
model is the IP Packet eXchange (IPX) network being mainly promoted by the commercial mobile communications industry. Basically, IPX is a telecommunications interconnection model for the exchange of IP based traffic between customers of separate mobile and fixed operators as well as other types of service provider (such as ISP), via IP based network-tonetwork interfaces and subject to end-to-end Service Level Agreements (SLAs) that add support for end-to-end QoS requirements [10]. Of note is that the option of using a sort of intervening packet-switched network was also identified in the Three-country Pilot (3CP) initiative [11] to deploy the TDMbased TETRA ISI interface, though it was discarded in favour of the use of leased lines due to ownership, responsibilities, and security issues of the intervening network that were critical at that time of decision making, in addition to the fact that the ISI interface under consideration was the TDM-based interface that favoured the use of E1 leased lines. However, when considering the deployment of IP-based ISI, the indirect interconnection option emerges as the most promising one for its benefits in terms of savings and scalability. An illustration of the direct and indirect IP interconnection cases is given in Figure 6.

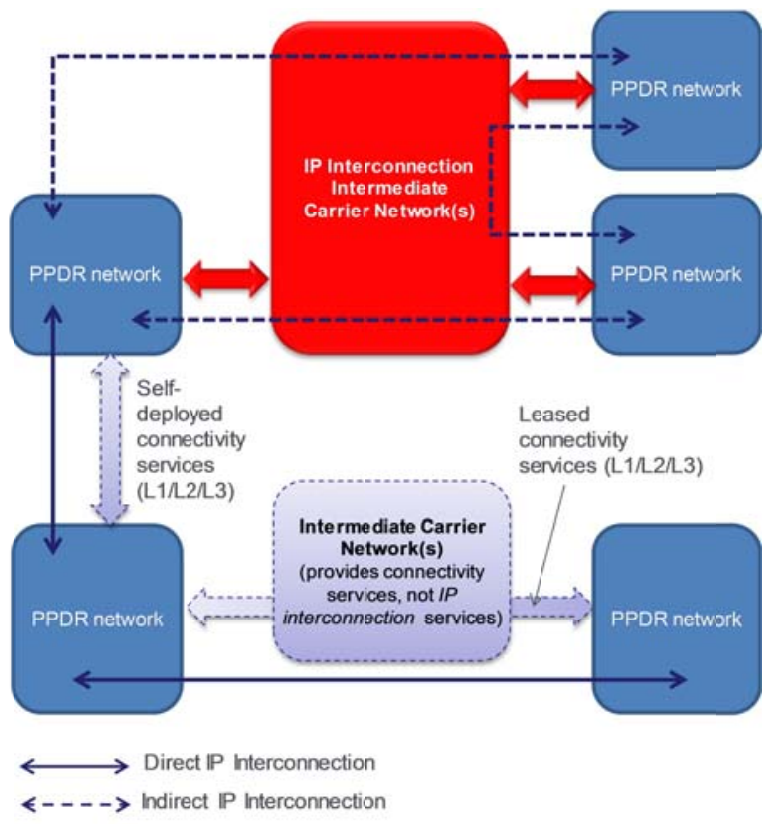

Fig. 6. Direct and indirect IP interconnection cases

\section{SUPPORT OF FUTURE BROADBAND SERVICES IN THE EUROPEAN ISI CLOUD NETWORK}

The adoption of IP as the basis for the evolution of an ISI protocol stack will also enable easy integration of current narrowband PPDR networks with broadband IP access technologies such as Wi-Fi and LTE. This integration will add high data rate capability to the PPDR systems.

Technological advances in the commercial domain have led to top-of-the-line radio technologies able to achieve performance levels close to Shannon's bound. The state of the art of commercial wireless technology evolution is Long Term Evolution (LTE) mobile broadband technology, currently positioned to be the dominant technology in future commercial mobile networks. LTE technology can provide a high bit rate, low latency IP connectivity service that can be used to deliver most of the new demanded PPDR data services [12]. The alignment to commercial technologies offers huge opportunities for creating and exploiting synergies between these two worlds, which have remained virtually separated to date. Using equipment developed for the mass market instead of niche products, the PPDR community will profit from the economy of scale, faster innovation and high competition between vendors. The same applies for the market of end user devices and dedicated software, where even stronger competition should be expected.

The adoption of LTE technology is gaining strong momentum among the PPDR community. National Public Safety Telecommunications Council (NPSTC), TETRA and Critical Communications Association (TCCA), European Telecommunications Standards Institute (ETSI) Technical Committee TETRA and other relevant organizations are backing LTE as the baseline technology for next generation broadband PPDR networks. At a standardization level, work is in progress at the 3rd Generation Partnership Project (3GPP), which is in charge of the LTE specifications, to develop enhancements to the LTE standard, such as support for deviceto-device and enhanced group communications with Push-toTalk (PTT) features, which will further increase the suitability of the LTE technology for PPDR and other professional sectors [13].

In this context, a plausible scenario for the interconnection of future PPDR networks is illustrated in Figure 7, where it is considered that current TETRA/TETRAPOL infrastructures could be complemented/upgraded/migrated with LTE/Wi-Fi broadband access, enabling also the case of roaming services for broadband access for data-centric/intensive applications.

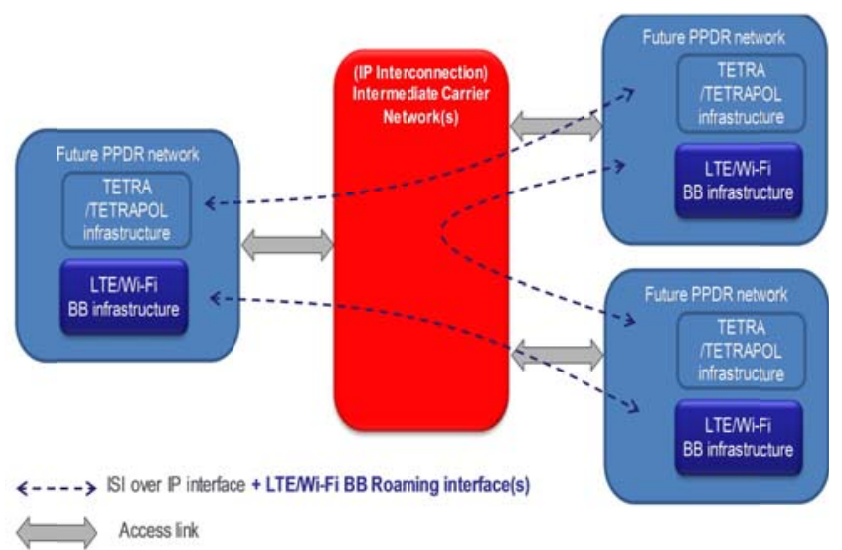

Fig. 7. Extension of the IP-based interconnection model to support future PPDR broadband roaming services 


\section{CONCLUSIONS AND FUTURE WORK}

ISITEP project pursues the achievement of operational interoperability among European first responders, addressing in a comprehensive manner the regulative, organizational, operational and technical level. ISITEP project offers a unique opportunity, since it is the first time that the main manufacturers of the PPDR European networks join end users and operators towards a common interconnection target. The European ISI Cloud Network, as part of the ISITEP framework, will enable PPDR TETRA/TETRAPOL national/regional infrastructures to be interconnected and to allow migration and communication services and associated applications within a secure framework.

This paper has described the key components for the realisation of the ISITEP cloud network, namely, (1) a new ISI over IP interface, (2) a variety of ISI gateways to account for the different TETRA/TETRAPOL interconnection scenarios as well as the use of legacy TETRA ISI E1 by some networks, (3) Security Gateways to provide confidentiality, integrity and intrusion detection and (4) the use of IP Interconnection services. Furthermore, the adoption of IP as the basis for the evolution of an ISI protocol stack also enables a smooth migration path from current narrowband PPDR networks towards the integration of forthcoming mobile broadband IPcentric access technologies such as Wi-Fi and LTE for PPDR communications.

Work is underway to finalise the specification of the new IP ISI and the development of the different ISI gateways. ISITEP specifications are intended to be subdued to the ETSI standard as Public Available Specification (PAS). Capabilities of the new ISI interface are planned to be demonstrated on the field and validated by end users and operators.

\section{ACKNOWLEDGMENT}

The research leading to these results has received funding from the European Union Seventh Framework Programme (FP7/2007-2013) THEME [SEC-2012.5.3-4]-[Global solution for interoperability between first responder communication systems - Integration Project] - "Inter System Interoperability for Tetra-TetraPol Networks" under grant agreement $n^{\circ}$ [312484]-[ISITEP].

\section{REFERENCES}

[1] G. Baldini, R. Ferrús, O. Sallent, Paul Hirst, Serge Delmas, Rafał Pisz, "The evolution of Public Safety Communications in Europe: the results from the FP7 HELP project", ETSI Reconfigurable Radio Systems Workshop, Sophia Antipolis, France, 12 December 2012

[2] Simon Forge, Robert Horvitz and Colin Blackman, "Study on use of commercial mobile networks and equipment for "mission-critical" highspeed broadband communications in specific sectors", Final Report, December 2014. Available online at https://ec.europa.eu/digitalagenda/en/news/use-commercial-mobile-networks-and-equipmentmission-critical-high-speed-broadband

[3] Becchetti, C.; Frosali, F.; Lezaack, E., "Transnational Interoperability: A System Framework for Public Protection and Disaster Relief," Vehicular Technology Magazine, IEEE, vol.8, no.2, pp.46,54, June 2013

[4] Etienne Lezaack (Editor), "Usage Candidate Scenarios", ISITEP Deliverable 2.1.2, November 2014. Available online at http://isitep.eu/

[5] EU Research Project on "Inter-system interoperablity for TETRATETRAPOL networks (ISITEP)". Project website: http://isitep.eu/

[6] ETSI EN 300 392-3-1, “TETRA V+D ISI General Design”, V1.3.1, August 2010
[7] ECMA 143 Private Integrated Services Network (PISN) - Circuit Mode Bearer Services - Inter-Exchange Signalling Procedures and Protocol (QSIG-BC)

[8] ECMA 133, Private Integrated Services Network (PISN) - Reference $\begin{array}{lllll}\text { Configuration for } & \text { PISN Exchanges } & \text { (PINX) }\end{array}$ 2nd edition (December 1998)

[9] 3GPP TR 22.893 V10.0.0 (2009-12), "Study into identification of advanced requirements for IP interconnection of services; (Release 10)", December 2009

[10] GSM Association, Official document IR.34, "Guidelines for IPX Provider networks (Previously Inter-Service Provider IP Backbone Guidelines), Version 9.1, May 2013. Available online at http://www.gsma.com/newsroom/wp-content/uploads/2013/05/IR.34v9.1.pdf

[11] ETSI TR 101 448, Version 1.1.1, "Functional requirements for the TETRA ISI derived from Three-Country Pilot Scenarios", May 2005

[12] Ferrús, R.; Sallent, O.; Baldini, G.; Goratti, L., "LTE: the technology driver for future public safety communications," Communications Magazine, IEEE, vol.51, no.10, pp.154,161, October 2013

[13] Ferrús, R.; Sallent, O., "Extending the LTE/LTE-A Business Case: Mission- and Business-Critical Mobile Broadband Communications," Vehicular Technology Magazine, IEEE, vol.9, no.3, pp.47,55, Sept. 2014 